\title{
Evaluating Home Health Care Nursing Outcomes With OASIS and NOC
}

\author{
Julia Stocker Schneider, Violet Barkauskas, Gail Keenan
}

Purpose: To determine the sensitivity and responsiveness of the Outcome and Assessment Information Set (OASIS) and the Nursing Outcomes Classification (NOC) to the effects of home healthcare nursing interventions.

Methods: A quasi-experimental before-after study was conducted using a sample of 106 home healthcare participants referred to one of seven participating Midwest home healthcare agencies for treatment of a cardiac condition. Patient outcomes data were collected at home healthcare admission and discharge using OASIS and NOC. Nursing intervention data were collected at each visit using the Nursing Interventions Classification (NIC). Intervention intensity was calculated by totaling the number of NIC interventions provided over the episode of care.

Findings: Neither OASIS nor NOC were sensitive to the effects of home healthcare nursing as measured by intervention intensity. The OASIS was not responsive to clinically discernable changes in patient outcomes; while the NOC was responsive to patient status change in the outcome categories including activities of daily living, cardiopulmonary status, coping, and illness management behavior.

Conclusions: Outcome measures that are more condition-specific and discipline-specific are more responsive to the effects of home healthcare nursing. Further research is needed to identify and refine outcome measures that are sensitive and responsive to the effects of nursing care in home health and other nursing settings.

Clinical Relevance: The use of outcome measures that are more sensitive and responsive to nursing are more effective in guiding nursing practice.

[Key words: home care, NOC, nursing-sensitive outcomes, OASIS, responsiveness, sensitivity]

Journal of Nursing Scholarship, 2008; 40:1, 76-82. (C)2008 Sigma Theta Tau International.

$\mathrm{N}$ ursing-sensitive outcomes have generated increased attention in recent years. Prominent examples of such studies include research that has linked nurse staffing to patient outcomes, and has identified nursingsensitive measures such as "failure to rescue" (Aiken, Clarke, Sloane, Sochalski, \& Silber, 2002; Needleman, Buerhaus, Mattke, Stewart, \& Zelevinsky, 2002). Consistent with the quest for nursing-sensitive outcome measures, the National Quality Foundation (NQF) recently developed a standardized set of nursing-sensitive outcomes using a consensus process to identify the most viable outcome measures for such a set (Kurtzman \& Kizer, 2005). Despite this heightened interest in nursing-sensitive outcomes, minimal consideration has been given to the psychometric properties that are crucial to successful outcomes' measurement, including instrument sensitivity and responsiveness.

This article is a report of a study in which investigators compared the sensitivity and responsiveness of OASIS to the Nursing Outcomes Classification (NOC) when used by nurses across seven Midwest home healthcare agencies.

\section{Background}

The OASIS is a 79-item instrument that was developed to provide for the standardized collection of outcomes data

Julia Stocker Schneider, RN, PhD, Lambda Zeta, Assistant Professor, University of Detroit Mercy, Detroit, MI; Violet Barkauskas, RN, PhD, FAAN, Rho, Associate Professor, University of Michigan, Ann Arbor, MI; Gail Keenan, RN, PhD, Rho, Associate Professor, University of Illinois, Chicago. Research was funded by the National Institute for Nursing Research (Grant F 31 NR07588-02), the Blue Cross Blue Shield Student Award Program (Grant 270.SAP), and the Michigan Nurses Association Competitive Research Award Program CURN Scholarship. Correspondence to Dr. Stocker Schneider, University of Detroit Mercy, 4001 W. McNichols Rd., Detroit, MI 48221; E-mail: stockju@udmercy.edu Accepted for publication October 26, 2007. 
in the home healthcare setting (Shaughnessy, Crisler, Hittle, \& Schlenker, 2002). Collecting outcomes data using OASIS is required by the Centers for Medicare and Medicaid Services (CMS) on all Medicare and Medicaid patients over the age of 18 who are receiving skilled home health nursing or therapy services, with the exception of pre- or postpartum services. The OASIS data set is used for outcomes-based quality improvement (OBQI), prospective pay, and public reporting of quality data through the home health compare initiative (CMS, 2006). Despite the fact that nursing care is the core service provided in home healthcare, this instrument has not yet been tested for sensitivity to nursing.

In addition to reliability and validity, outcome measures must show sensitivity and responsiveness. Though these terms have been used interchangeably in the literature, they are distinct properties. Sensitivity refers to the ability of an instrument to detect patients' change in status that can be attributed to receiving interventions (Liang, 2000; Mark \& Salyer, 1999). Responsiveness can be further differentiated as the ability of an outcomes measure to detect clinically meaningful change in a patient's state (Liang, 2000). The effectiveness of home healthcare nursing interventions on patient outcomes cannot be adequately measured without the use of sensitive and responsive outcome instruments. Though OASIS has been tested extensively and used routinely in home healthcare since 1999 (Shaughnessy et al., 2002), it has not yet been evaluated explicitly for sensitivity and responsiveness to nursing.

The most comprehensive effort to date to identify and refine outcomes sensitive to nursing has been the development of the Nursing Outcomes Classification (NOC). The NOC contains 330 outcomes that allow measurement of results of nursing care (Moorhead, Johnson, \& Maas, 2004). Though the NOC was designed purposefully for measuring nursing-sensitive outcomes, it has not yet been tested sufficiently for sensitivity and responsiveness.

This research was conducted to address a critical gap in knowledge regarding the sensitivity and responsiveness of outcome measures to the effects of nursing interventions in home healthcare services. The specific aims of the study were to: (a) compare the sensitivity of selected components of OASIS to selected NOC outcomes in detecting differences in outcomes among cardiac patients who received different intensities of home health care nursing interventions as measured by NIC when selected patient factors were controlled; and (b) compare the responsiveness of corresponding measures in OASIS and NOC in detecting clinically discernable changes in outcomes among heart disease patients receiving home health nursing care.

\section{Methods}

Patients referred to one of seven participating Midwest home healthcare agencies for treatment of a primary cardiac condition between September 2002 and July 2003 were re- cruited for the study. All 106 participants received intermittent skilled home health nursing care, and were recruited by home healthcare RNs who were trained as data collectors for the study. Though some patients received other supportive services (e.g., physical therapy, home health aide services) in conjunction with home health nursing care, the predominant service provided to all study participants was nursing. Patients under age 18 and pre- and postpartum patients were excluded, because OASIS was not designed for use with these populations (Shaughnessy et al., 2002).

\section{Instruments}

OASIS and NOC were compared using seven outcome categories shown in Table 1 . These outcome areas were selected according to outcome measures identified by the OASIS cardiac condition quality indicator group (Shaughnessy et al., 1994). During the study design phase, the OASIS items were grouped into outcome categories according to their conceptual similarity by home healthcare nurse experts in a focus group including: (a) activities of daily living (ADLs), (b) cardiopulmonary status, (c) coping, and (d) instrumental activities of daily living (IADLs). The experts subsequently selected NOC outcomes to correspond with each of the designated outcome categories. Additional outcome categories deemed important by the nurse experts for cardiac patients in home health care were added including: (a) effects of medication, (b) illness management behavior, and (c) knowledge. These additional outcomes reflect outcomes routinely monitored and considered important by home healthcare nurses.

OASIS was developed by a multidisciplinary team as a way to improve home healthcare quality (Shaughnessy et al., 2002). The instrument includes core and conditionspecific outcome measures that were identified using criteria such as clinical meaningfulness, importance, reliability, and representation across dimensions of health status (Shaughnessy et al., 1994). All 14 OASIS outcome measures used in the study were ordinal, with a range of three to five choices per item. Validity and reliability of OASIS has been established (kappa range $=0.44-0.91$, mean 0.59-0.60; Shaughnessy et al., 2002), with global measures indicating better statistical properties compared to condition-specific measures (Madigan \& Fortinsky, 2000).

A nursing-sensitive patient outcome is defined in NOC as "an individual, family, or community state, behavior, or perception that is measured along a continuum in response to nursing intervention(s)" (Moorhead et al., 2004, p. 26). Each NOC outcome consists of a label, definition, and indicators. NOC labels and indicators are measured according to a 5-point Likert scale. One NOC was selected for the study for each of the seven outcome categories noted in Table 1. Validity and reliability have been established for NOC across the continuum of care (Keenan et al., 2003a, 2003b; Maas et al., 2002; Moorhead et al., 2004). Because previous studies have indicated that NOC label scores 
Table 1. Study Outcome Categories Including Cardiac Condition, OASIS Items, and Corresponding and Additional NOC Outcomes

\begin{tabular}{|c|c|c|}
\hline Outcome categories & OASIS outcome items & NOC outcomes \\
\hline Activities of daily living (ADLs) & $\begin{array}{l}\text { M0640 grooming } \\
\text { M0650 ability to dress upper body } \\
\text { M0660 ability to dress lower body } \\
\text { M0670 bathing } \\
\text { M0680 toileting } \\
\text { M0690 transferring } \\
\text { M0700 ambulation/locomotion } \\
\text { M0710 feeding or eating }\end{array}$ & 0300 self care: activities of daily living (ADL) \\
\hline Cardiopulmonary status & M0490 dyspnea & 0400 cardiac pump effectiveness \\
\hline Coping & M0580 anxiety level & 1302 coping \\
\hline Effects of medication & a & 2301 medication response \\
\hline $\begin{array}{l}\text { Instrumental activities of daily } \\
\text { living (IADLs) }\end{array}$ & $\begin{array}{l}\text { M0720 preparing meals } \\
\text { M0740 laundry } \\
\text { M0750 housekeeping } \\
\text { M0780 management of oral medications }\end{array}$ & $\begin{array}{l}0306 \text { self care: instrumental activities } \\
\text { of daily living (IADL) }\end{array}$ \\
\hline IIIness management behavior & a & 1609 treatment behavior: illness or injury \\
\hline Knowledge & a & 1824 knowledge: illness care \\
\hline
\end{tabular}

andicates no OASIS item(s) for this category.

are sufficiently reliable, NOC label scores only were measured in this study (Keenan et al., 2003a, 2003b). Nursing interventions provided by home healthcare nurses were measured using NIC. NIC incorporates interventions provided by nurses across practice settings. Nursing interventions are defined in NIC as "any treatment, based upon clinical judgment and knowledge, that a nurse performs to enhance patient/client outcomes" (McCloskey Dochterman \& Bulechek, 2004, p. xxiii). Each NIC intervention includes a distinguishable definition and list of related activities. Validity has been established for NIC, and it has undergone more rigorous testing than have other existing measures of nursing treatments in all settings. Field-testing has been conducted (McCloskey et al., 2004), yet reliability of NIC intervention selection has not been reported.

For this study, the home healthcare nurses selected NICs performed at each home visit from a list categorized according to outcome. The list included 21-50 NIC interventions per outcome category (mean 38), and included only those NICs judged by home healthcare nurse experts to be pertinent to that outcome. For example, NICs frequently selected to improve the effects of medication outcome included: Medication Management, Vital Signs Monitoring, and Teaching: Prescribed Medication. Intervention intensity was determined by totaling the number of interventions provided to each participant during the episode of care according to outcome category. Though this method did not allow weighting specific NICs by importance, it was a baseline estimate for comparing the overall intervention intensity. Each NIC intervention could be selected only once per visit per outcome category, but could be selected for more than one category when relevant.
A global estimate of change was used as an external measure of responsiveness for OASIS and NOC. In the absence of a "gold standard," construct validity for clinically relevant change can be established by comparing independent ratings by patients and providers on such a general health measure (Deyo \& Centor, 1986; Deyo \& Patrick, 1995). For this study, the participants and nurses were asked at the discharge visit to independently estimate whether change in overall health status and knowledge, coping, and selfmanagement of the health condition had occurred. Each rating had a 5-point Likert scale ranging from much better, to much worse. The overall health rating was used to indicate intra-individual patient change in physical health status, including cardiopulmonary status and related factors including the effects of medication and ADL status. Global health measures were used because they indicate the status of the main health problem. In this case, cardiopulmonary status, and the closely intertwined effectiveness of cardiac medications and functional status were seen as related to the global health measure. A knowledge, coping, and self-management rating was used as a criterion for intra-individual change in coping, IADL status, knowledge, and illness management behavior outcomes.

\section{Procedure}

A quasi-experimental, before-after design was used for this study. In order to determine instrument sensitivity of OASIS and NOC to the effects of home health care nursing interventions, OASIS and NOC data were collected on admission (pre-test measure) and discharge (post-test measure) for each participant for the selected outcome areas. NIC intervention data were gathered, according to outcome category for which the interventions were intended to improve at 
each visit by home healthcare nurses. Intervention intensity was calculated for each category by totaling the number of NIC interventions performed over the episode of care. Sensitivity was determined by assessing the relationship of the change in OASIS and NOC scores to the intensity of nursing interventions for each outcome category when other factors were controlled. Responsiveness was determined by comparing OASIS and NOC change scores to the presence of clinically discernable change as shown by agreement of independent ratings by nurses and the patients indicating the presence of change.

The participating home healthcare agencies included two visiting nurse associations, two hospital-based agencies, two private nonprofit agencies, and one proprietary agency. The agencies ranged in size from 4,310 to 136,524 annual visits (median 16,701), and employed 8 to 60 full-time registered nurse equivalents (median 10). Home healthcare RN volunteers were recruited from each of the seven sites to collect data on eligible patients who consented to participate. The $\mathrm{RN}$ participants were required to attend two 1.5 hour education sessions, to be oriented to the study procedure including NIC selection and NOC rating. In order to improve reliability, rules for NIC intervention selection were established, and NIC and NOC competency exercises were included. Periodic joint visits were made by the principal investigator (PI) in order to measure interrater reliability.

Following completion of the education sessions, participating home health care RNs recruited patients referred for admission to the home healthcare agency for treatment of a cardiac condition. For patients who consented to participate in the study, the RNs completed the OASIS instrument during the admission visit. Additionally, the RNs completed NOC ratings for the comparable outcome measures, and selected NIC interventions to describe the nursing care provided for the outcomes being measured. The NIC data were collected at each subsequent $\mathrm{RN}$ visit according to the care provided. All home visits for the study participants were provided by RNs who had completed the research training for the study. Though most visits were provided by the primary RN, occasional visits were provided as needed by other RNs who were trained for the study. On the discharge visit, RNs completed the nurse global estimate of change measures, and the final OASIS and NOC measures. Then, the RNs asked patients to independently and anonymously rate the global estimate of change measures. If patients were not discharged within 60 days of home health enrollment, the OASIS and NOC data were obtained at 60 days according to CMS OASIS collection requirements. If patients were rehospitalized without returning home promptly, if patients died, or if patients refused further services, the nurses completed the OASIS and NOC tools for the most recent visit.

\section{Findings}

Patients in the study ranged in age from 25-97 years $($ mean $=77) ; 51 \%$ of the participants were male, and $95 \%$ were Caucasian. Asian, Black, and Hispanic patients each made up approximately $1 \%$ of the sample. The primary diagnoses for admission to home care were coronary artery disease (CAD; 36\%), congestive heart failure (CHF; 31\%), and other cardiac diagnoses $(33 \%)$ including acute myocardial infarction, atrial fibrillation, intermediate coronary syndrome, mitral stenosis, angina, aortic valve disorder, and cardiomyopathy. Many participants with CAD underwent coronary artery bypass surgery before being admitted to home care.

Sample participants had a mean of 3.42 comorbidities $(S D=1.52)$. Top comorbidities included essential hypertension $(41 \%)$, diabetes $(29 \%)$, and cardiac dysrythmia $(20 \%)$. The majority of participants $(48 \%)$ lived with a spouse or significant other, while $28 \%$ lived alone, and $19 \%$ lived with other family members. Assistance was received from another person residing in the home $(66 \%)$, outside the home $(65 \%)$, both $(35 \%)$, and paid help $(6 \%)$. The majority of the participants received various forms of assistance from a caregiver, including IADL assistance $(70 \%)$, psychosocial support $(69 \%)$, environmental support $(66 \%)$, support for participation in appropriate medical care $(60 \%)$, and ADL support $(34 \%)$. Participants received a mean of $9.19 \mathrm{RN}$ visits $(S D=4.7)$, remained on service for 28 days $(S D=14)$, and had a mean total $\mathrm{RN}$ visit time of 7.15 hours $(S D=3.7)$ for the home healthcare episode.

The PI conducted periodic joint visits with the RNs, in order to estimate the reliability of the study measures. At these joint visits, the $\mathrm{RN}$ and the PI independently rated the measures according to the study procedure. Using these data, intraclass correlations (ICC) were calculated. The ICC reliability of the number of NICs selected per outcome category $(n=52)$ ranged from $0.27-0.63(M=0.48)$. ICC reliability for OASIS $(n=16)$ ranged from $0.35-0.83(M=0.65)$. The NOC ICC reliability $(n=16)$ ranged from $0.39-0.97$ $(M=0.66)$.

\section{Sensitivity}

Outcome change scores were determined for OASIS and NOC by calculating the difference between admission and discharge scores for each item. To allow comparison, OASIS and NOC change scores were standardized by converting them into $z$-scores. Risk adjustment was accomplished by including additional OASIS variables in the regression model that were theoretically sound and statistically significant, including reason for discharge, agency, caregiver assistance with ADLs, and primary diagnosis severity (Tables $2 \& 3$ ). For outcome categories without a corresponding OASIS measure, the multiple regressions were the analyses (Table 2). For these outcomes, the measure of sensitivity was statistical significance in the relationship between intervention intensity and NOC outcomes change when risk factors were controlled.

As shown in Table 2, the $p$-values for intervention intensity exceeded 0.05 , showing that the outcomes were not sensitive to intervention intensity. For the remaining 
Table 2. Regression Tables for Intervention Intensity and Outcome Change for NOC Only Measures $(n=106)$

\begin{tabular}{lccc}
\hline Outcome category & $R^{2}$ for model & $\begin{array}{c}\text { Intervention intensity } \\
(p \text {-values })\end{array}$ & $\begin{array}{c}\text { Reason for discharge } \\
(p \text {-values })\end{array}$ \\
\hline Effects of medication & 0.089 & 0.601 & $0.002^{*}$ \\
Illness management behavior & 0.161 & 0.971 & $0.018^{*}$ \\
Knowledge & 0.000 & 0.914 & - \\
\hline
\end{tabular}

Note. ${ }^{*} p \leq 0.05$

outcome categories, a repeated measures analysis of variance (ANOVA) was conducted (Table 3). The measure of sensitivity was the interaction between the test (OASIS or NOC) and intervention intensity; the lack of statistical significance achieved for these outcome categories indicated that neither OASIS nor NOC were sensitive to the effects of the home health care nursing interventions.

\section{Responsiveness}

A receiver-operating characteristic (ROC) curve was constructed for each of the seven outcome categories to determine if OASIS and NOC were responsive to clinically discernable change. The criterion for the discernable change in an outcome area was agreement on independent estimate of change ratings by the patient and $\mathrm{RN}$. To make the criterion stronger, cases that lacked agreement in perceptions of change between patient and nurse were excluded, because the occurrence of clinically discernable change was not as apparent in these cases where both estimators did not perceive the change. Also, estimate of change data could not be collected on 12 participants who did not receive a discharge visit because of rehospitilization or refusal of further services. Thus, analyses were based on 94 cases for estimate of change in overall health status, and 80 cases for estimate of knowledge, coping, and self-management.

The ROC curve is the true positive rate (sensitivity) and the true negative rate (specificity) for designated cut points in change score (Deyo \& Centor, 1986). The two cut points used in this analysis were patient and nurse agreement that (a) the patient was much better (maximum positive change), and (b) the patient was better or the same (minimal or no change). These cut points were chosen, because estimate of change data were not available for participants who demonstrated a decline in health status (because no discharge visit occurred if the patient was rehospitalized or died).

The area under the ROC curve denotes the global measure of accuracy for an instrument and can be interpreted as the probability of correctly identifying patients who have clearly improved and those who have not. A larger area indicates greater accuracy when significance is achieved (Deyo \& Centor, 1986). As shown in Table 4, OASIS was not statistically significant in any of the outcome categories, indicating that OASIS was not responsive to the effects of home care nursing interventions. For NOC, statistical significance $(p=0.05)$ was achieved for ADLs, cardiopulmonary status, coping, and illness management behaviors, but not for effects of medication, IADLs, and knowledge. The most responsive outcome category for NOC was coping ( area $=0.760)$, followed by ADLs (area=0.734), illness management behavior (area $=0.726)$ and cardiopulmonary status $(0.655)$.

\section{Discussion}

Sensitive outcome measures can show change that results from receiving interventions. In this study, neither OASIS nor NOC showed significant sensitivity to the effects of nursing interventions. Reliability issues (specifically with the intervention intensity as measured by number of NICs) likely contributed to these results. Though the NIC is in routine use in many practice settings, reliability for the NIC has not been sufficiently established. The reliability of NIC interventions could be dramatically improved by providing some simple rules for assigning the more global versus narrower NIC interventions as well as devising a weighting scheme for NIC. Despite this limitation, the current

Table 3. Sensitivity of Outcome Change to Intervention Intensity Using Repeated Measures ANOVA ( $N=106)$

\begin{tabular}{lccccc}
\hline Outcome category & $\begin{array}{c}\text { Test } \\
\text { (OASIS or NOC) }\end{array}$ & $\begin{array}{c}\text { Intervention } \\
\text { intensity }\end{array}$ & $\begin{array}{c}\text { Interaction } \\
\text { between test and NIC }\end{array}$ & $\begin{array}{c}\text { Reason } \\
\text { for discharge }\end{array}$ & $\begin{array}{c}\text { Caregiver } \\
\text { assistance w/ ADLs }\end{array}$ \\
\hline ADLs & 0.684 & 0.192 & 0.717 & $0.001^{*}$ & $0.000^{*}$ \\
Cardiopulmonary status & 0.175 & 0.909 & 0.119 & - & - \\
Coping & 0.993 & 0.791 & 0.585 & - & - \\
IADLS & 0.127 & 0.513 & 0.317 & $0.001^{*}$ & $0.012^{*}$ \\
\hline
\end{tabular}

Note. ${ }^{*} p \leq 0.05$. 
Table 4. ROC Curve Analysis of Responsiveness of OASIS and NOC to Intervention Intensity

\begin{tabular}{|c|c|c|c|c|}
\hline Outcome category & OASIS area & OASIS significance ( $p$-value) & NOC area & NOC significance ( $p$-value) \\
\hline$A D L s^{a}$ & 0.577 & 0.332 & 0.734 & $0.003^{*}$ \\
\hline Cardiopulmonary status ${ }^{a}$ & 0.645 & 0.067 & 0.655 & $0.052^{*}$ \\
\hline Coping $^{b}$ & 0.614 & 0.249 & 0.760 & $0.008^{*}$ \\
\hline Effects of medication ${ }^{\mathrm{a}}$ & - & - & 0.621 & 0.128 \\
\hline IADLs ${ }^{b}$ & 0.683 & 0.063 & 0.609 & 0.269 \\
\hline IIIness management behaviors ${ }^{b}$ & - & - & 0.726 & $0.022^{*}$ \\
\hline Knowledge $^{b}$ & - & - & 0.689 & 0.055 \\
\hline
\end{tabular}

Note. ${ }^{a}$ Agreement on health status change used as criterion $(n=80) .{ }^{\mathrm{b}}$ Agreement on knowledge, coping, and management of health used as criterion $(n=76) . * 0.05$.

study extends previous research on nursing outcomes, because most investigators to date have measured nursing care according to $\mathrm{RN}$ resource use (e.g., number of $\mathrm{RN}$ visits in home care or RN staffing ratios in hospital settings), rather than by specific nursing interventions provided. Measuring specific nursing interventions and their effects on patient outcomes can help determine nursing contributions to patient care and allow identification of best practices.

Outcome measures must also be responsive, meaning that such instruments indicate change when clinically relevant change has occurred, and show no change when clinically discernable change is absent. This study showed that NOC was more responsive than was OASIS. The NOC is more condition-specific, which contributes to its superior responsiveness to OASIS. Though generic outcome measures (such as OASIS) demonstrate utility across health conditions, the findings of this study indicate the limitations of generic outcome measures in effectively measuring clinically relevant change for specific conditions. Outcome measures that are more specific to the condition and service provided are more likely to capture relevant change that results from the interventions provided. Such properties are essential for determining and advancing best practices in nursing.

The implementation of OASIS in home healthcare settings has positively affected overall home healthcare quality by providing a framework for outcomes-based quality improvement. Given the volume of home healthcare services provided by nurses, it is important that outcome measures used in home healthcare are sensitive and responsive to nursing. As indicated in this study, OASIS did not show certain outcomes deemed important by home healthcare nurse experts in the care of cardiac patients at home, including effects of medication, knowledge, and illness management behavior. In fact, home healthcare nurses provided more interventions per episode of care to address illness management behavior ( $M=46.32$ NICs per episode; $S D=22.43)$, effect of medication $(M=40.10 ; S D=20.29)$, and knowledge outcomes $(M=36.24 ; S D=18.91)$, than they did for IADL $(M=34.19 ; S D=22.07)$, coping $(M=23.75 ; S D=20.96)$, or ADL $(M=23.26 ; S D=17.39)$ outcomes.

As expected, the most home health nursing interventions were provided for cardiopulmonary status $(M=46.32$; $S D=22.43$ ), though dyspnea is the only cardiopulmonary outcome included in OASIS. Because patients with cardiac disease have the highest home care utilization rate outcome measures should show changes that result from the care received by this patient population.

\section{Limitations}

Despite data collection at multiple sites throughout a Midwestern state and attempts to recruit minority populations, the ethnic diversity of the sample was limited. Also, the findings are not generalizable to patients whose health status declined because of death or rehospitilization, because they were excluded from the analysis. Some bias might have resulted in the global estimate of change, because of the desire of patients and nurses to show improvement resulting from care, though the methodology used has shown validity as a criterion measure. Lack of adjustment for receipt of home health care from other healthcare professionals (e.g., physical therapists) was also a limitation, though the effects of nursing can be better isolated in the home care setting than in most other settings where nurses practice.

Because of difficulty in recruiting participants, a smaller than anticipated sample size was obtained, that likely contributed to insufficient interrater reliability findings. Other factors, such as the need for further clarity in selecting more global versus narrower NIC interventions influenced reliability findings. One can anticipate that improved reliability would have resulted from the addition of further training and experience by nurses in the use of NIC and NOC.

Finally, the use of an intervention count to measure intervention intensity has certain limits, because all NIC interventions do not contribute equally to the achievement of outcomes. However, this study extends the current outcomes measurement science by showing measurement of nursing care more precisely than has been done previously. The naturalistic design of this study helps to enhance generalizability of the findings, because the researchers measured nursing care as it was delivered in practice, rather than imposing controlled conditions.

\section{Conclusions}

While sensitivity is difficult to measure, further research is important in home health care and other nursing settings, 
in order to further identify and refine outcome measures that truly reflect patients' change that results from nursing care provided. Responsiveness should also be considered in outcomes measurement. As shown in this research, conditionspecific and discipline-specific measures (such as NOC) are more likely to indicate higher responsiveness than are more generic measures (such as OASIS). Finally, outcome tools should include measures that show care that has been provided. According to the findings of this study, OASIS did not indicate outcomes that directly resulted from care provided in several areas including effects of medication, illness management behavior, and knowledge. The use of such intermediate outcomes that directly reflect care provided by home care nurses would be more useful to nurses in practice and could better elucidate best practices.

\section{Clinical Resources}

Center for Nursing Classification \& Clinical Effectiveness (NIC/NOC)

http://www.nursing.uiowa.edu/about_us/nursing_know-

ledge/clinical_effectiveness/index.htm

Outcome and Assessment Information Set (OASIS)

http://www.cms.hhs.gov/OASIS/

National OASIS Integrity Project

http://www.fazzi.com/Whats\%20New/oasis_integrity.htm

Medicare Quality Improvement Community: Home Health

http://www.medqic.org/

\section{References}

Aiken, L.H., Clarke, S.P., Sloane, D., Sochalski, J., \& Silber, J.H. (2002). Hospital nurse staffing and patient mortality, nurse burnout, and job dissatisfaction. JAMA, 288(16), 1987-1993.
Centers for Medicare and Medicaid Services. (2006). Home health quality initiatives. Retrieved August 8, 2006, from http://www.cms.hhs.gov/ HomeHealthQualityInits/

Deyo, R.A., \& Centor, R.M. (1986). Assessing the responsiveness of functional scales to clinical change: An analogy to diagnostic test performance. Journal of Chronic Disease, 39(11), 897-906.

Deyo, R.A., \& Patrick, D.L. (1995). The significance of treatment effects: The clinical perspective. Medical Care, 33(4), 286-291.

Keenan, G., Barkauskas, V., Stocker, J., Johnson, M., Maas, M., Moorhead, S., et al. (2003a). Establishing the validity, reliability, and sensitivity of NOC in an adult care nurse practitioner setting. Outcomes Management, 7(2), 74-83.

Keenan, G., Barkauskas, V., Stocker, J., Johnson, M., Maas, M., Moorhead, S., et al. (2003b). Establishing the validity, reliability, and sensitivity of NOC in home care settings. Journal of Nursing Measurement, 11(2), 135-155.

Kurtzman, E.T., \& Kizer, K.W. (2005). Evaluating the performance and contribution of nurses to achieve an environment of safety. Nursing Administration Quarterly, 29(1), 14-23.

Liang, M.H. (2000). Longitudinal construct validity: Establishment of clinical meaning in patient evaluative instruments. Medical Care, 38(9, Suppl. II), II-84-II-90.

Maas, M., Reed, D., Reeder, K.M., Kerr, P., Specht, J., Johnson, M., et al. (2002). Nursing Outcomes Classification: A preliminary report of field testing. Outcomes Management, 6(3), 112-119.

Mark, B.A., \& Salyer, J. (1999). Methodological issues in treatment effectiveness and outcomes research. Outcomes Management in Nursing Practice, 3(1), 12-18.

Madigan, E.A., \& Fortinsky, R.H. (2000). Additional psychometric evaluation of the outcomes and assessment information set (OASIS). Home Health Care Services Quarterly, 18(4), 49-61.

McCloskey Dochterman, J.M., \& Bulechek, G.M. (Eds.). (2004). Nursing Iinterventions classification (4th ed.). St. Louis, MO: Mosby.

Moorhead, S.A., Johnson, M., \& Maas, M. (Eds.). (2004). Nursing outcomes classification (3rd ed.). St. Louis, MO: Mosby.

Needleman, J., Buerhaus, P., Mattke, S., Stewart, M., \& Zelevinsky, K. (2002). Nurse-staffing levels and the quality of care in hospitals. New England Journal of Medicine, 346(22), 1715-1722.

Shaughnessy, P.W., Crisler, K.S., Hittle, D.F., \& Schlenker, R.E. (2002). OASIS and outcome-based quality improvement in home health care: Research and demonstration findings, policy implications, and considerations for future change. Denver, CO: Center for Health Services Research.

Shaughnessy, P.W., Schlenker, R.E., Crisler, K.S., Powell, M.C., Hittle, D.F., Kramer, A.M., et al. (1994). Vol. 1 Final report: Measuring outcomes of home health care. Denver, CO: Center for Health Policy Research. 